\title{
Incidence and management of condylar resorption after orthognathic surgery: An overview
}

Selene Barone ${ }^{\mathrm{a}}$ (1) Giorgio Cosentini ${ }^{\mathrm{b}}$ Francesco Bennardo ${ }^{\mathrm{a}}$ Alessandro Antonelli ${ }^{\mathrm{a}}$ Amerigo Giudice ${ }^{\mathrm{a}}$ (1)

${ }^{a}$ Department of Health Sciences, School of Dentistry, Magna Graecia University of Catanzaro, Catanzaro, ltaly

${ }^{b}$ Department of Health Sciences, Magna Graecia University of Catanzaro, Catanzaro, ltaly
Objective: Condylar resorption (CR) is one of the major post-surgical complications of orthognathic surgery. This systematic review (SR) aimed to evaluate epidemiological data, risk factors, and therapeutical management of CR. Methods: Six databases were screened by two investigators until September 2020 to obtain all SRs. After reading the titles and abstracts, eligible SRs were determined and data extraction was performed. Using the latest version of A Measurement Tool to Assess Systematic Reviews, the methodological quality of the included SRs was determined. Results: Ten SRs with low or criticallylow methodological quality were included in this review. Mandibular hypoplasia on the sagittal plane and hyperdivergent growth pattern on the vertical plane were the most common skeletal alterations in which CR could occur after orthognathic surgery. Post-operative condylar changes were analyzed both on two-dimensional and three-dimensional (3D) radiographic examinations. The incidence of CR was not related to the fixation method. Based on the severity of the pathological conditions, management of CR can include conservative or surgical therapy. Conclusions: Despite the limited evidence in literature, CR is considered a consequence of orthognathic surgery. However, an accurate diagnosis of $\mathrm{CR}$ and a better orthognathic surgical planning must include 3D radiographic examinations to improve pre- and post-surgical comparison. Welldesigned studies with long-term follow-up and 3D data are needed to clarify the findings of this analysis..

[Korean J Orthod 2022;52(1):29-41]

Key words: Three-dimensional diagnosis and treatment planning, Class 111 orthognathic surgery, Temporomandibular joint, Condylar resorption

Received July 15, 2021; Revised September 14, 2021; Accepted September 24, 2021.

Corresponding author: Amerigo Giudice.

Professor, Department of Health Sciences, School of Dentistry, Magna Graecia University of Catanzaro, Viale Europa - 88100 Catanzaro, ltaly.

Tel +39-0961712469 e-mail a.giudice@unicz.it

How to cite this article: Barone S, Cosentini G, Bennardo F, Antonelli A, Giudice A. Incidence and management of condylar resorption after orthognathic surgery: An overview. Korean J Orthod 2022;52:29-41.

(C) 2022 The Korean Association of Orthodontists.

This is an Open Access article distributed under the terms of the Creative Commons Attribution Non-Commercial License (http://creativecommons.org/licenses/by-nc/4.0) which permits unrestricted non-commercial use, distribution, and reproduction in any medium, provided the original work is properly cited. 


\section{INTRODUCTION}

For patients undergoing orthognathic surgery, several surgical risks with different severity rates must be considered.' Condylar resorption (CR) is an uncommon but well-known clinical condition that can occur after surgical repositioning of the jaws. ${ }^{2,3}$ It represents the advanced stage of condylar remodeling, irreversibly affecting the temporomandibular joint (TMJ). ${ }^{3-5}$ As a result of mechanical load, the adaptive mechanisms that normally allow to balance bone resorption and bone formation on the condylar surface are completely lost, resulting in morphological alterations of the condylar structure. ${ }^{5,6}$ Microstructural changes include a significantly reduced condylar volume and a pathological deformed profile on the condylar surface. ${ }^{7-9}$ External changes have a significant influence on aesthetics and may include reduced posterior facial height, mandibular retrusion, and anterior open bite, especially if bilateral CRs occur. ${ }^{7,10}$

Based on the etiology, CR can be distinguished into primary and secondary CR. ${ }^{8}$ Primary $\mathrm{CR}$ is diagnosed when CR exists without a well-known cause. ${ }^{8,11}$ Conversely, both local (trauma, inflammation, infection, etc.) and systemic (rheumatic immune pathologies, steroid therapy, etc.) agents can be etiological factors of secondary CR. ${ }^{6}$ Referring to orthognathic surgery, all osteotomies can lead to $\mathrm{CR}$, which can occur in both monomaxillary and bi-maxillary surgery. ${ }^{2,12}$ Bilateral sagittal split osteotomy (BSSO) and/or Le Fort 1 osteotomy can modify the mechanical forces on the condylar surface, resulting in $\mathrm{CR} .{ }^{12}$

Several studies have focused on condylar alterations after orthognathic surgery, and some systematic reviews (SRs) aimed to determine the most crucial risk factors for CR. However, the different methodological approaches do not allow to draw firm deductions. The rationale of this overview was to summarize the most recent knowledge on CR after orthognathic surgery (CROS), emphasizing the limitations of previous studies in order to critically evaluate their conclusions and identify the gaps in knowledge that should be filled by future researches with adequate protocols.

This study aimed to systematically review all SRs and meta-analyses (MAs) to analyze CROS in order to provide evidence-based information about epidemiological data, risk factors, and management of CR, evaluating each outcome in correlation with the qualitative methodological analysis of the included reviews.

\section{MATERIALS AND METHODS}

All SRs were systematically reviewed according to the Population, Intervention, Comparison, Outcomes, and Study criteria to assess CR in patients who underwent orthognathic surgery.

\section{Search strategy}

Six databases were investigated: PubMed, Cochrane Library, Google Scholar, Scopus, LILACS, and Web of Science. The electronic search was conducted until September 2020, using keywords and MeSH terms connected by the Boolean operator "AND." The PubMed search combined the following term sequence: "orthognathic surgical procedures"[Mesh] AND "condylar resorption" AND "systematic review" and "condylar resorption" AND "orthognathic surgery" AND "systematic review." The other search databases combined the following term sequence: "orthognathic surgery" AND "condylar resorption" AND "systematic review." Additionally, a manual search was conducted in the reference lists of the selected SRs. No restriction of language or publication date was imposed.

\section{Review selection}

The electronic search was independently conducted by two investigators (SB and GC), screening titles and abstracts in parallel to evaluate the reviews for eligibility. In case of missing information, full-text reading was necessary for a final decision. A third author (AG) discussed and resolved any discrepancies between the two authors. SRs and MAs were included, which allowed for the extraction of data on CROS. Narrative reviews, overview of reviews, duplicate articles, studies with no evaluation of condylar morphology, and studies on orthognathic surgery of syndromic patients or cleft lip and palate patients were excluded. The Cohen's kappa coefficient $(\kappa)$ was calculated to determine the inter-rater agreement between the two investigators (SB and GC).

\section{Data extraction}

The data extraction from the eligible reviews was independently performed by the same two investigators ( $\mathrm{SB}$ and $\mathrm{GC}$ ), recording the following information: author, publication date, study design (SR or MA), number of included studies, number of included patients, dentoskeletal malocclusion, type of intervention, methodological data, quality assessment of primary studies, outcomes, results of reviews, and author's conclusion.

\section{Assessment of methodological quality}

The methodological quality of each SR was independently assessed by the two investigators (SB and GC) using the latest version of A Measurement Tool to Assess Systematic Reviews (AMSTAR-2). ${ }^{13}$ AMSTAR-2 includes 16 domains, 9 non-critical items, and 7 critical items that strongly influence the final score. The quality assessment can range from high to critically low. ${ }^{13}$ 


\section{RESULTS}

\section{Search results and review selection}

A total of 62 records were selected from the electronic search of six different databases (PubMed, $\mathrm{n}=9$; Cochrane Library, $n=0$; Google Scholar, $n=25$; Scopus, $n=13$; LILACS, $n=1$; Web of Science, $n=14$ ), and no studies were added by manual search. After excluding

Table 1. List of excluded studies

\begin{tabular}{ll}
\hline \multicolumn{1}{c}{ Study } & Reason of exclusion \\
\hline Al-Moraissi, 2016 & Incoerent topic \\
Al-Moraissi, 2017 & Incoerent topic \\
Al-Ryhami, 2009 part 1 & Incoerent topic \\
Al-Ryhami, 2009 part 2 & Incoerent topic \\
Catherine, 2016 & Incoerent topic \\
Francisco, 2020 & Overview \\
Haas Junior, 2019 & Overview \\
Ji, 2020 & Incoerent topic \\
Kersey, 2003 & Incoerent topic \\
Merhaban, 2020 & Incoerent topic \\
Nicolielo, 2017 & Incoerent topic \\
Romero, 2019 & Incoerent topic \\
Sansare, 2015 & Incoerent topic \\
Sonego, 2014 & Incoerent topic \\
Verlinden, 2015 & Incoerent topic \\
\hline
\end{tabular}

the duplicates, 25 potentially significant studies were found. After screening titles and abstracts, 23 full-text articles were screened for eligibility, and 2 studies were excluded. ${ }^{5,14}$ After full-text reading, 13 reviews were excluded because they did not meet the inclusion criteria (Table 1). Figure 1 shows the flow diagram of the search strategy and SR selection: 10 SRs were included for the qualitative analysis. ${ }^{7,8,15-22}$ Quantitative analysis could not be conducted because no MAs were performed. The inter-rater agreement coefficient was $\kappa=0.93$.

\section{Data extraction}

Table 2 summarizes all data extracted from the SRs. Each SR included a different number of studies (ranging from 6 to 76), and none performed an MA. The publication year ranged from 2008 to 2019. Regarding the study design, most of the SRs included observational retrospective and prospective studies. Non-randomized controlled trial (non-RCT) was the most common study type, with the others being case control study, cohort study, case series, and case report. ${ }^{7,8,15-22}$ Only two SRs described the results of RCTs. ${ }^{19,20}$ Dentoskeletal Class 11 was the most frequent malocclusion analyzed in the included SRs. ${ }^{7,8,15-22}$ Other studies evaluated patients with dentofacial malformations such as skeletal Class 111, skeletal asymmetry, and skeletal open bite. , $^{7,20,21}$ The most common surgical procedure included BSSO with or without Le Fort 1 osteotomy. ${ }^{7,16-22}$ Five reviews reported the outcomes after Le Fort 1 osteotomy alone, unilateral sagittal split osteotomy, and intraoral vertical ramus osteotomy. ${ }^{7,16-18,22}$ Post-surgical changes of con-

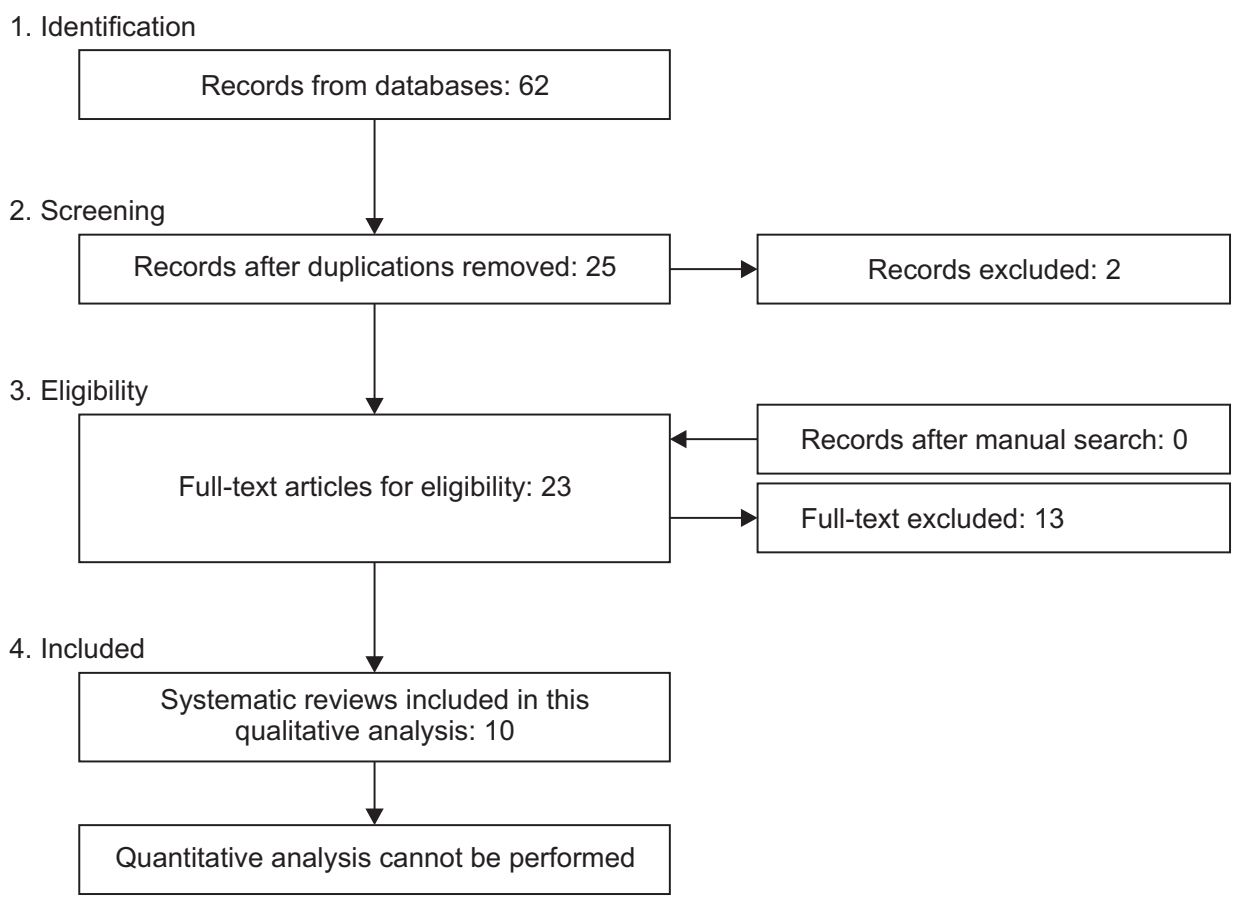

Figure 1. A flowchart of the search strategy and selection of systematic reviews. 


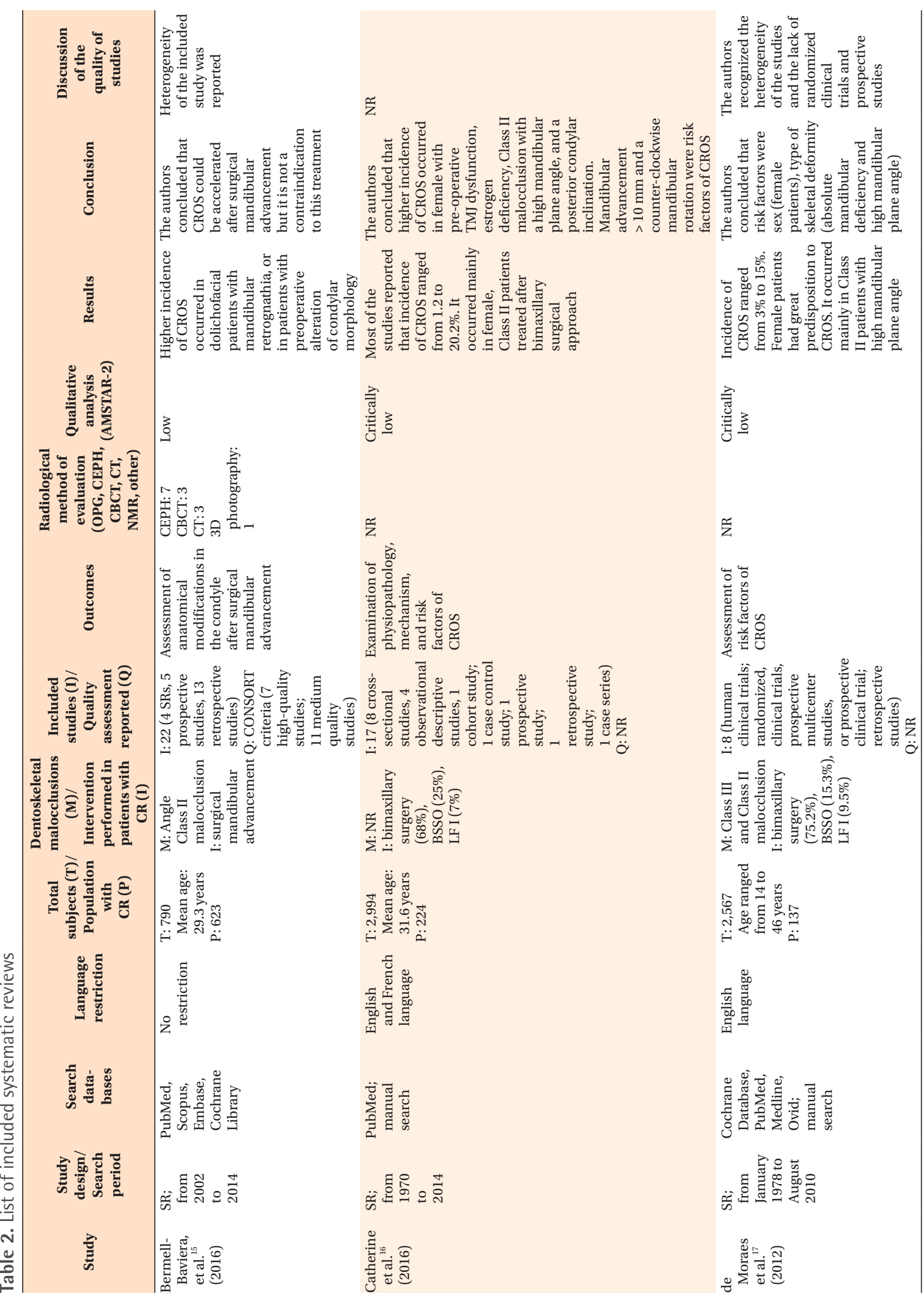




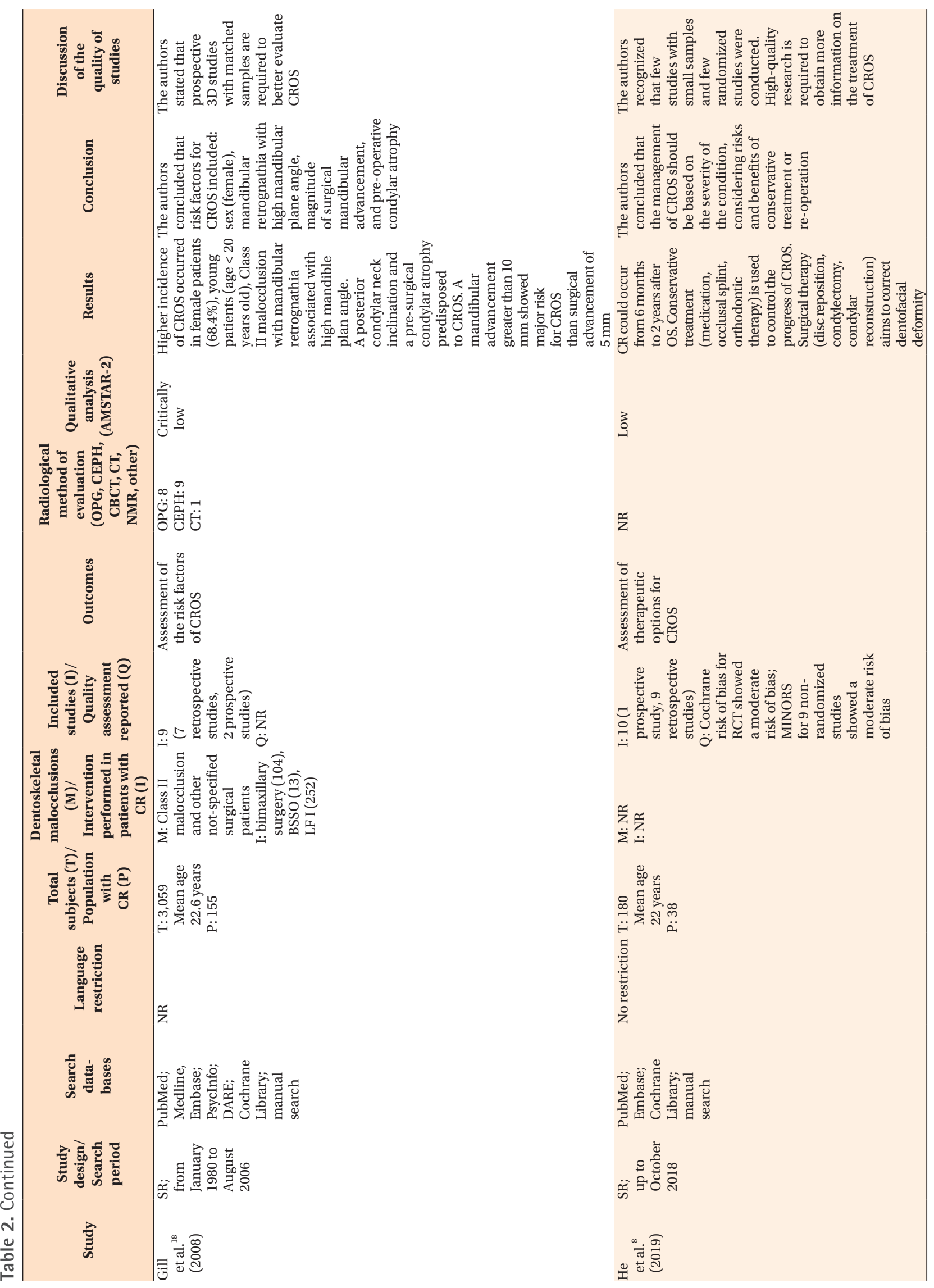




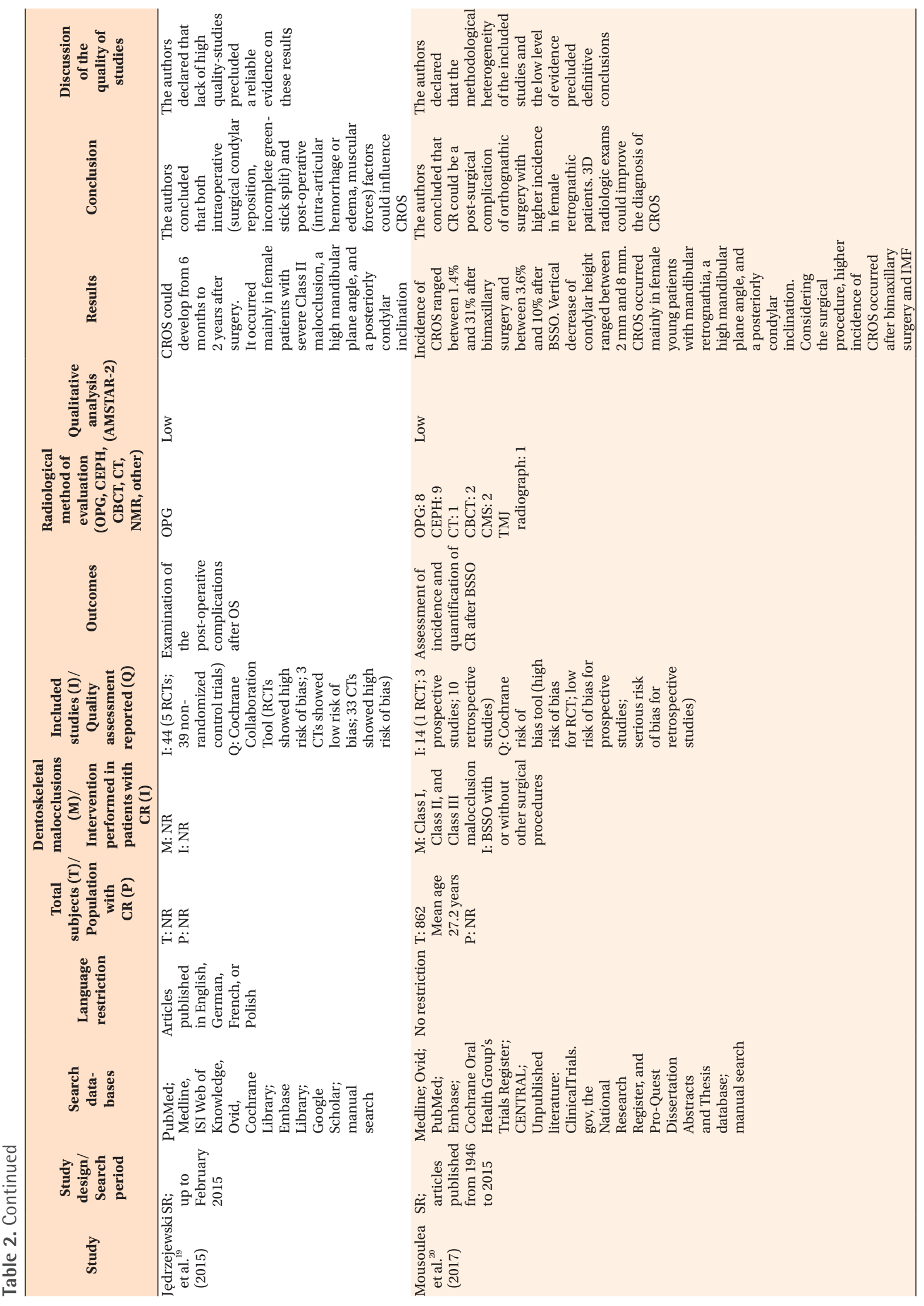




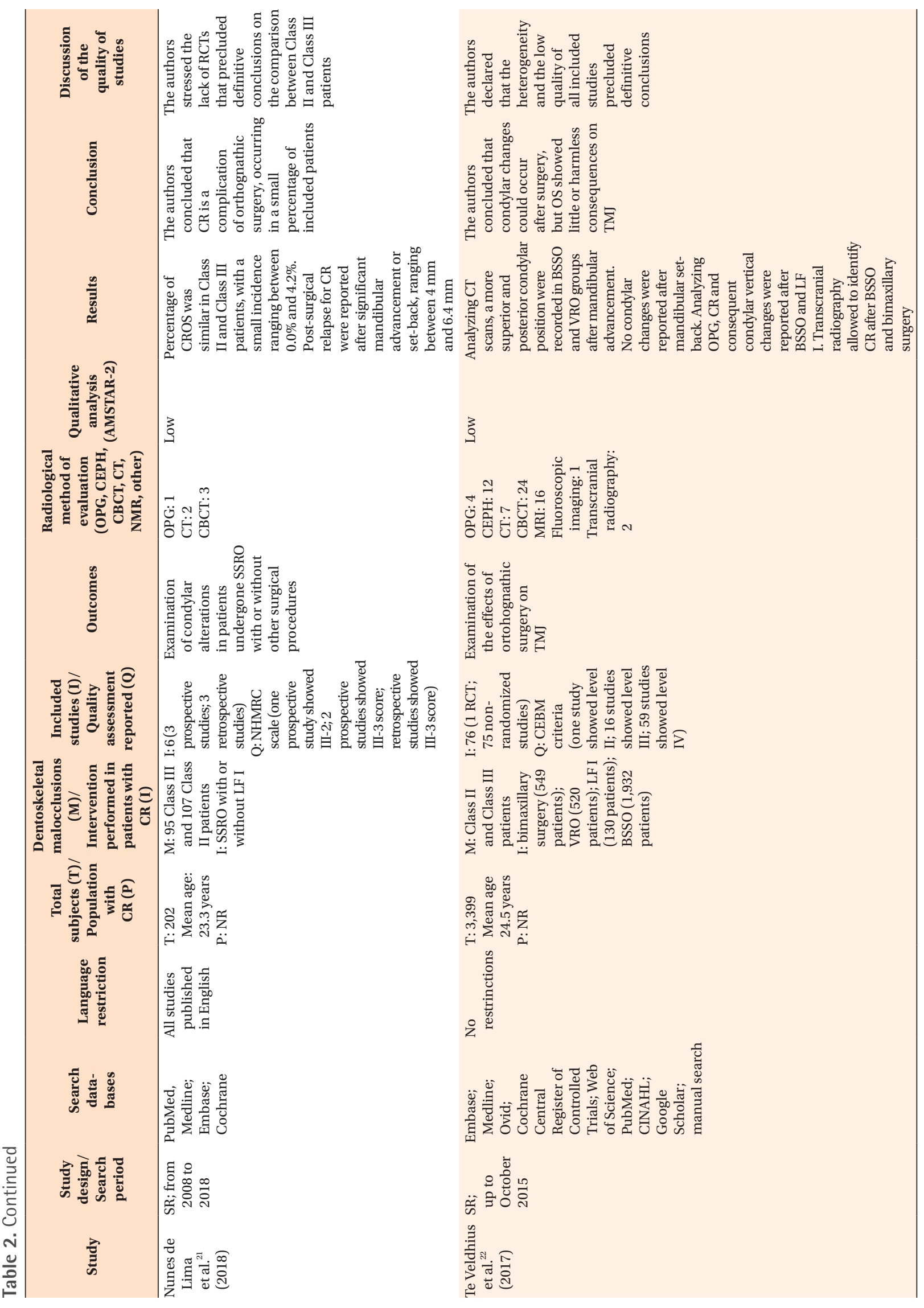




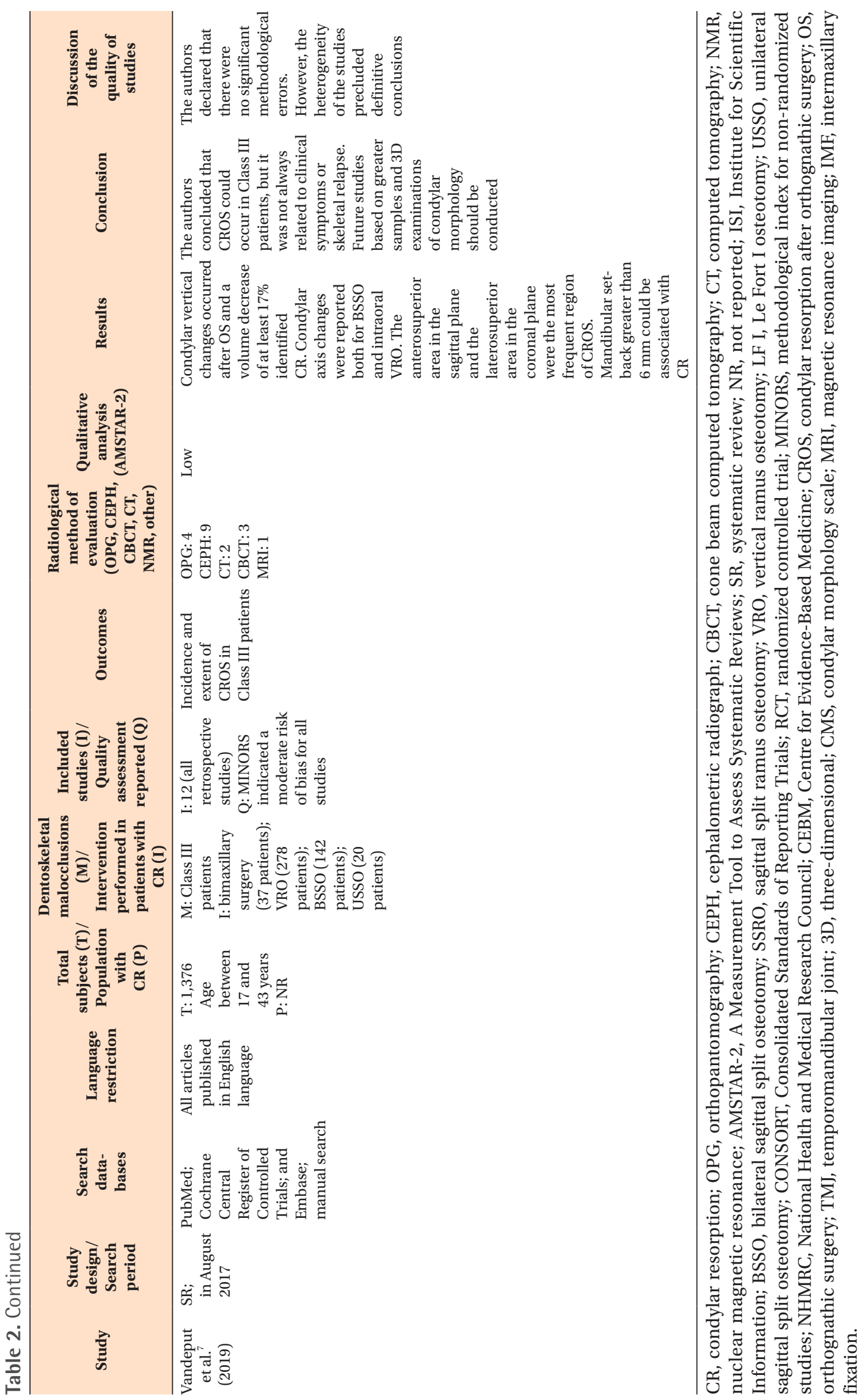


dylar morphology were analyzed on two-dimensional (2D) radiographic examinations (orthopantomography and/or lateral cephalogram), three-dimensional (3D) radiographic examinations (computed tomography, cone beam computed tomography, and magnetic resonance imaging), or combined 2D and 3D examinations. ${ }^{7,8,15-22}$ The follow-up period ranged between 12 and 60 months, with a minimum and a maximum follow-up period of 3 and 192 months, respectively. ${ }^{7,8,15-22}$ The number of patients included in the SRs ranged between 180 and 3,777 , with a mean age of 26.6 years. ${ }^{7,8,15-22}$ Post-orthognathic CR was the primary outcome in the included studies evaluating the morphological changes of the mandibular condyle after surgery. ${ }^{7,15-22}$ Two SRs reported the effectiveness of $\mathrm{CR}$ management. ${ }^{8,16}$

\section{Analysis of methodological quality}

The methodological quality of each SR was determined using AMSTAR-2. ${ }^{13}$ Most of the included SRs had a low methodological quality. ${ }^{7,8,15,19-22}$ Three SRs had a critically low methodological quality. ${ }^{16-18}$ The most critical items of the AMSTAR-2 checklist were the absence of details for excluded studies and the absence of a comprehensive search strategy.

\section{Condylar resorption}

Nine of the included SRs aimed to describe the main characteristics and risk factors of CROS (Table 3). ${ }^{7,15-22}$ Jędrzejewski et al. ${ }^{19}$ reported that CR manifestations usually ranged from 6 months to 2 years after surgical treatment, but Catherine et al. ${ }^{16}$ extended this time to 6 years post-operatively. Although with different diagnostic methods, CR could be identified by both radiographic and clinical signs. ${ }^{8,16,20}$ Further, CR was recognized by $2 \mathrm{D}$ radiological examinations if there was a reduction of ramus or condylar height, with a minimum vertical decrease of $2 \mathrm{~mm} .{ }^{20}$ A 3D analysis of the condylar profile assessed that a reduction of at least 17\% of the condylar volume was considered as the cut-off value for the diagnosis of CR. ${ }^{8}$ Clinical signs of bilateral CR included an anterior open bite or a Class 11 malocclusion with retrognathia. ${ }^{16}$ On the contrary, an active process of unilateral CR could identify mandibular asymmetry with an ipsilateral Class 11 malocclusion and a contralateral open bite. $^{16}$

There is no agreement among the included SRs about the role of sex or age in CROS., ${ }^{715-22}$ Five SRs reported that females showed a higher risk for CR than males, with an approximate female-to-male ratio of $5: 1 .^{16,17,19-21}$

Table 3. Summary of the incidence of condylar resorption after orthognathic surgery

\begin{tabular}{|c|c|}
\hline Question & Condylar resorption after orthognathic surgery \\
\hline Who & $\begin{array}{l}\text { Patients undergoing orthognathic surgery (from } 1.2 \% \text { to } 31 \% \text { ) } \\
\text { Sex: } \\
\text { - 5F:1M } \\
\text { Surgical treatment: } \\
\text { - Bimaxillary surgery: from } 67.8 \% \text { to } 75.2 \% \text { of cases; } \\
\text { - BSSO: from } 15.3 \% \text { to } 24.5 \% \text { of cases; } \\
\text { - Le Fort I osteotomy: from } 6.7 \% \text { to } 9.5 \% \text { of cases } \\
\text { Fixation method: } \\
\text { - Rigid fixation (from } 6.3 \% \text { to } 13 \% \text { of cases); } \\
\text { - Wire fixation ( } 9.9 \% \text { of cases) }\end{array}$ \\
\hline What & $\begin{array}{l}\text { Radiological signs: } \\
\text { - A vertical decrease of } 2 \mathrm{~mm} \text { or more of the ramus or the condylar height; } \\
\text { - A reduction of at least } 17 \% \text { of the condylar volume } \\
\text { It was estimated that } 60 \% \text { of cases with CROS developed a resorption that ranged between } 10 \text { and } 19 \% \text { of the } \\
\text { condylar surface, } 30 \% \text { of cases showed CROS ranging between } 20 \text { and } 29 \% \text {, and } 10 \% \text { of cases had CROS greater } \\
\text { than } 30 \% \text {. }\end{array}$ \\
\hline When & From 6 months to 6 years after surgery \\
\hline Where & Antero-superior and latero-superior areas of the condylar head \\
\hline Why & $\begin{array}{l}\text { CROS was correlared with the following risk factors: } \\
\text { - Impaction of the maxilla and counter-clockwise rotation of the upper occlusal plane with a more posterior } \\
\text { position of the condyles ( } 87 \% \text { of the cases); } \\
\text { - Mandibular hypoplasia with high mandibular plane angle }(21.8 \% \text { of cases) and with normal/low plane angle } \\
\text { ( } 2.2 \% \text { of cases); } \\
\text { - Pre-surgical signs of condylar atrophy }(20-44 \% \text { of cases); } \\
\text { - Presurgical TMD recognized ( } 24 \% \text { of cases) }\end{array}$ \\
\hline
\end{tabular}

F, female; M, male; BSSO, bilateral sagittal split osteotomy; CROS, condylar resorption after orthognathic surgery; TMD, temporomandibular disorder. 
Two SRs stated that young patients may be more prone to $\mathrm{CR}$, while de Moraes et al. ${ }^{17}$ concluded that age could not be correlated with post-operative CR. ${ }^{16,17,21}$ In terms of pre-surgical evaluation, it was mandatory to exclude an active process of condylar atrophy, the presence of severe temporomandibular disorders, and an intra-articular inflammatory damage., $86-18$

Risk factors for CR were evaluated both in the sagittal and vertical planes. ${ }^{7,15-22}$ Angle Class 11 with mandibular hypoplasia was the most common malocclusion in which CR occurred after a significant mandibular advancement (incidence rate: $1.4-31 \%) .^{7,15-22}$ An orthognathic plan with advancements greater than $10 \mathrm{~mm}$ may be a risk factor for CR. ${ }^{16}$ Furthermore, in Angle Class 11 malocclusions, micrognathia often involved condylar morphology characterized by a small volume, a reduced adaptive capacity to external load, and an increased risk of CR. ${ }^{8}$ Only one SR reported a more prone CR in Angle Class 111 malocclusion after a surgical mandibular setback greater than $6 \mathrm{~mm}^{8}{ }^{8}$ On the vertical plane, a hyperdivergent skeletal pattern with an anterior open bite, a low posterior-to-anterior facial height, and an increased mandibular plane angle (MPA) could cause CR., ${ }^{75-22}$ However, the entity to define the critical clockwise rotation of the mandible was not unanimously accepted, but it could be described when the MPA was greater than $40^{\circ} .^{16,18}$ In patients with a dolichofacial profile, a presurgical posterior condylar inclination was exposed to CR because the antero-superior surface and the laterosuperior area were subjected to an excessive load on the sagittal and coronal planes, respectively. ${ }^{7,25}$

\section{Fixation method}

Six of the included SRs evaluated the type of fixation in relation to $C R^{8,16-19,21}$ Wire fixation, rigid fixation (bicortical miniscrew and miniplates), and intermaxillary fixation (IMF) are reported as the possible options. ${ }^{16,17}$ The incidence of CROS or surgical relapse was not affected by the fixation method in $6.3-13 \%$ of the cases. ${ }^{16,17,21}$ However, a majority of the authors recorded a high risk of CROS after a prolonged IMF rather than a rigid fixation, while no difference was found between wire and rigid fixation. ${ }^{8,18,19}$ Although no studies have compared the two types of rigid fixation, Jędrzejewski et al. ${ }^{19}$ reported that bicortical miniscrews could determine inferior alveolar nerve damage.

\section{Management of condylar resorption}

Two included SRs reported the main therapeutic approaches for $\mathrm{CR}^{8,16}$ Despite the fact that there are no precise guidelines on the management of CR to date, several suggestions were made, differentiating the preand post-surgical phases of evaluation. ${ }^{8}$ In the preoperative phase, the aim was to annul or limit the risk factors. ${ }^{8}$ In the post-surgical phase, the objectives included the treatment of inflammation and pain, as well as the correction of skeletal deformities and occlusal instability., ${ }^{816}$ Conservative or surgical treatment were proposed. ${ }^{8,16}$ Conservative therapy managed to avoid CR progression, stabilizing the pathologic conditions without improving dentofacial deformities. ${ }^{8,16}$ It included anti-inflammatory drugs, occlusal splints, physiotherapy, and in some cases, orthodontic or restorative treatments. ${ }^{8,16}$ Surgical treatment should be performed at least 6 months after orthognathic surgery, considering the severity of $C R$, the surgeon's experience, and the patient's intentions. ${ }^{8}$ Disc repositioning, condylectomy, chondro-costal graft, or complete prosthetic TMJ reconstruction were more invasive therapeutic options that were reported as possible treatments for $\mathrm{CR}^{8,16}$

\section{DISCUSSION}

The purpose of this review was to examine the current evidence of CROS, relating the methodological analysis of each SR included in this study. CR has been recognized as one of the major post-surgical complications occurring in orthognathic patients. ' It can be considered an irreversible correspondence of the physiological condylar remodeling, characterized by a severe impairment of condylar morphology with or without clinical symptoms. ${ }^{5}$ In this overview, a population of patients with skeletal deformities who had undergone orthognathic surgery was analyzed, excluding the SRs of syndromic or cleft lip and palate patients in whom different heterogeneous comorbidities can be associated with skeletal malformations. ${ }^{23}$ This SR aimed to answer the following questions: "Can CR be considered a severe complication of orthognathic surgery?;" "What are the main risk factors for CROS?;" and "How can CROS be managed better?"

Ten SRs were included in this overview. ${ }^{7,8,15-22}$ Quantitative analysis could be reported because no MAs were performed. ${ }^{7,815-22}$ For each SR, the qualitative analysis recorded the final score of AMSTAR-2 obtained by the evaluation of its 16 items. ${ }^{13}$ Although the absence of MA precluded the evaluation of some critical domains, the methodological assessment of the included SRs ranged between low and critically-low quality. ${ }^{7,815-22}$ A comprehensive search strategy (item 4) and adequate details for the excluded studies (item 7) were the most common missing data in the AMSTAR-2 checklist.

The qualitative evaluation of the included SRs allowed to analyze the main characteristics of CROS, summarizing the most significant risk factors and better management reported in literature. CR related to orthognathic surgery can occur 6 months to 6 years after the surgical treatment, and both clinical and radiological signs 
should be examined. It was not unanimously accepted if sex or age could influence this pathological condition. However, young female patients seem to be more prone to CROS. ${ }^{7,15-22}$ Despite the limited evidence in literature, altered estrogen levels could interfere with the post-operative morphological changes occurring on the condylar surface. ${ }^{24}$ An increased inflammatory process negatively involves the synovial tissue and fibrocartilage synthesis, predisposing to TMJ instability and bone resorption. ${ }^{25}$ CR may expose the patient to skeletal relapse, resulting in an anterior open bite and a Class 11 dental relationship for mandibular clockwise rotation if it occurs bilaterally. ${ }^{16}$ Similarly, unilateral CR could be recognized by a contralateral open bite and an ipsilateral Class 11 malocclusion, emphasizing an asymmetric profile of the mandible. ${ }^{16}$ These clinical manifestations are consequent to $\mathrm{CR}$ because suitable joint relationships are required to allow mandibular functions, even with altered condylar morphology. ${ }^{21}$

Despite the low quality of evidence, all SRs identified Angle Class 11 malocclusion as the most frequent skeletal deformity in which CROS occurs. ${ }^{7,15-22}$ Surgical treatment of mandibular hypoplasia aimed to obtain a stable advancement of the jaw without post-operative relapse. An orthognathic plan with a mandibular advancement greater than $10 \mathrm{~mm}$ should be considered with caution because it represents a risk factor for CR. ${ }^{16}$ After surgery, backward suprahyoid muscle forces and reduced condylar volume are the most common problems in Class 11 patients, which decrease the adaptive capacity to external load, favor irreversible condylar alterations, and predispose to relapse. ${ }^{21,25,26}$

Despite the low qualitative assessment of the included SRs, hyperdivergent facial type was recorded as a significant risk factor for CROS on the vertical plane., ${ }^{75-22}$ Concerning the clockwise rotation of the mandible, the specific cut-off for CR was not unanimously accepted, but an MPA greater than $40^{\circ}$ was reported as a critical value. ${ }^{16,18}$ Major condylar changes were emphasized in patients with a lower posterior-to-anterior facial height ratio due to a significant increase in perimandibular tissue stretching after surgery. ${ }^{27}$ Furthermore, hyperdivergent facial type is often associated with a pre-operative posterior inclination of the condyle. ${ }^{7,15-22} \mathrm{~A}$ large surgical advancement of the mandible with an important counter-clockwise rotation causes excessive forces on the antero-superior and latero-superior areas of the condylar head, predisposing to CROS because unbalanced load occurs on less dense surfaces. ${ }^{8,20}$

Referring to the management of CR, two SRs described the main treatment options for CROS. ${ }^{8,16}$ A conservative therapeutic approach aimed to stop the pathologic progression, stabilizing the clinical conditions with anti-inflammatory drugs, occlusal splint, physiotherapy, and orthodontic or restorative treatments. ${ }^{8,16,27}$ Surgical therapy is reserved for severe cases of CROS, and it should be performed no sooner than 6 months after orthognathic surgery. ${ }^{8,28}$ The goal of the surgical options is to improve the clinical situation, correct the post-operative relapse, and restore an adequate condylar morphology. ${ }^{8,16,25}$ However, a reoperation should be considered with caution, evaluating the pathological progression, and should be meticulously planned to avoid worsening of the disease. ${ }^{8}$

In this overview, the main limitation of the conclusions relates to the presence of SRs with low or critically-low methodological quality. All reported results should be interpreted considering an imprecise search strategy of the studies and a limited methodological assessment of CROS. Most of the SRs included studies that evaluated CR on 2D radiographs, but 3D analysis of condylar profile is fundamental to obtain more specific information about the trend and severity of $\mathrm{CR} .^{7,20,29,30}$ As reported in literature, a reduction of at least $17 \%$ of the condylar volume was considered a significant radiological sign of active $\mathrm{CR}^{7,8,30}$ To date, orthognathic surgery has been a precise surgical procedure with a pre-operative 3D planning. ${ }^{14}$ An accurate pre-surgical 3D morphometric analysis of the condyles could improve the treatment plan and avoid post-operative relapses, allowing to maximize safe movements and minimize risky ones. The extent of the jaws' movements, direction, and rotational component could be influenced by the pre-surgical condition of the condyle by evaluating the possible degree of compromise. For this reason, the pre-operative assessment of condylar morphology could also be performed on 3D scans, excluding an active process of condylar atrophy or intra-articular inflammation. Because a post-surgical 3D examination was often required, a pre-operative and post-operative comparison of the condylar surface could be achieved, allowing to recognize important details for the diagnosis and entity of CROS. ${ }^{7,30}$

\section{CONCLUSION}

$\mathrm{CR}$ is a possible consequence of orthognathic surgery, with an incidence rate of $1-31 \%$. Mandibular deficiency, female hyperdivergent patients, and pre-surgical condylar alterations were the most reported risk factors for CROS. To limit inflammatory processes and improve clinical conditions, an adequate therapeutic approach involves conservative or surgical treatment, considering the severity of $C R$ and disease progression. Although many advancements have been made with the application of 3D technologies, it is still difficult to find a standardized method for intraoperative condylar repositioning. To date, scientific evidence is scarce and limited in order to draw certain conclusions. Future perspectives 
must include improvements in both the diagnostic and prognostic phases. An accurate diagnosis must be based on 3D data after an adequate standardization of the radiological characteristics of $\mathrm{CR}$ has been defined. Additionally, well-designed RCTs with long-term follow-up and pre- and post-operative 3D comparisons of condylar surfaces should be implemented to confirm the findings of this review.

\section{CONFLICTS OF INTEREST}

No potential conflict of interest relevant to this article was reported.

\section{REFERENCES}

1. Pelo S, Saponaro G, Patini R, Staderini E, Giordano A, Gasparini G, et al. Risks in surgery-first orthognathic approach: complications of segmental osteotomies of the jaws. A systematic review. Eur Rev Med Pharmacol Sci 2017;21:4-12.

2. Jung S, Choi Y, Park JH, Jung YS, Baik HS. Positional changes in the mandibular proximal segment after intraoral vertical ramus osteotomy: surgeryfirst approach versus conventional approach. Korean J Orthod 2020;50:324-35.

3. Ma RH, Li G, Yin S, Sun Y, Li ZL, Ma XC. Quantitative assessment of condyle positional changes before and after orthognathic surgery based on fused 3D images from cone beam computed tomography. Clin Oral Investig 2020;24:2663-72.

4. Hsu LF, Liu YJ, Kok SH, Chen YJ, Chen YJ, Chen $\mathrm{MH}$, et al. Differences of condylar changes after orthognathic surgery among Class 11 and Class 111 patients. J Formos Med Assoc 2021. doi: 10.1016/ j.jfma.2021.01.018. [Epub ahead of print]

5. Francisco 1, Guimarães A, Lopes M, Lucas A, Caramelo F, Vale F. Condylar form alteration on skeletal class 11 patients that underwent orthognathic surgery: an overview of systematic reviews. J Clin Exp Dent 2020;12:e695-703.

6. Catherine Z, Breton P, Bouletreau P. Management of dentoskeletal deformity due to condylar resorption: literature review. Oral Surg Oral Med Oral Pathol Oral Radiol 2016;121:126-32.

7. Vandeput AS, Verhelst PJ, Jacobs R, Shaheen E, Swennen G, Politis C. Condylar changes after orthognathic surgery for class 111 dentofacial deformity: a systematic review. Int J Oral Maxillofac Surg 2019;48:193-202.

8. He Z, Ji H, Du W, Xu C, Luo E. Management of condylar resorption before or after orthognathic surgery: a systematic review. J Craniomaxillofac Surg 2019;47:1007-14.
9. Claus JDP, Koerich L, Weissheimer A, Almeida MS, Belle de Oliveira R. Assessment of condylar changes after orthognathic surgery using computed tomography regional superimposition. Int J Oral Maxillofac Surg 2019;48:1201-8.

10. Lee JY, Lee SM, Kim SH, Kim Yl. Long-term follow-up of intersegmental displacement after orthognathic surgery using cone-beam computed tomographic superimposition. Angle Orthod 2020;90:548-55.

11. Ji YD, Resnick CM, Peacock ZS. Idiopathic condylar resorption: a systematic review of etiology and management. Oral Surg Oral Med Oral Pathol Oral Radiol 2020;130:632-9.

12. NiÑo-Sandoval TC, Almeida RAC, Vasconcelos BCDE. Incidence of condylar resorption after bimaxillary, Lefort 1, and mandibular surgery: an overview. Braz Oral Res 2021;35:e27.

13. Shea BJ, Reeves BC, Wells G, Thuku M, Hamel C, Moran J, et al. AMSTAR 2: a critical appraisal tool for systematic reviews that include randomised or non-randomised studies of healthcare interventions, or both. BMJ 2017;358:j4008.

14. Haas Junior OL, Guijarro-Martínez R, de Sousa Gil AP, da Silva Meirelles L, Scolari N, Muñoz-Pereira $\mathrm{ME}$, et al. Hierarchy of surgical stability in orthognathic surgery: overview of systematic reviews. Int J Oral Maxillofac Surg 2019;48:1415-33.

15. Bermell-Baviera A, Bellot-Arcís C, Montiel-Company JM, Almerich-Silla JM. Effects of mandibular advancement surgery on the temporomandibular joint and muscular and articular adaptive changes-a systematic review. Int J Oral Maxillofac Surg 2016;45:545-52.

16. Catherine Z, Breton P, Bouletreau P. Condylar resorption after orthognathic surgery: a systematic review. Rev Stomatol Chir Maxillofac Chir Orale 2016;117:3-10.

17. de Moraes PH, Rizzati-Barbosa CM, Olate S, Moreira RW, de Moraes M. Condylar resorption after orthognathic surgery: a systematic review. Int J Morphol 2012;30:1023-8.

18. Gill DS, El Maaytah M, Naini FB. Risk factors for post-orthognathic condylar resorption: a review. World J Orthod 2008;9:21-5.

19. Jędrzejewski M, Smektała T, Sporniak-Tutak K, Olszewski R. Preoperative, intraoperative, and postoperative complications in orthognathic surgery: a systematic review. Clin Oral Investig 2015;19:96977.

20. Mousoulea S, Kloukos D, Sampaziotis D, Vogiatzi T, Eliades T. Condylar resorption in orthognathic patients after mandibular bilateral sagittal split osteotomy: a systematic review. Eur J Orthod 2017;39: 294-309. 
21. Nunes de Lima V, Faverani LP, Santiago JF Jr, Palmieri C Jr, Magro Filho 0, Pellizzer EP. Evaluation of condylar resorption rates after orthognathic surgery in class 11 and 111 dentofacial deformities: a systematic review. J Craniomaxillofac Surg 2018;46:66873.

22. Te Veldhuis EC, Te Veldhuis AH, Bramer WM, Wolvius EB, Koudstaal MJ. The effect of orthognathic surgery on the temporomandibular joint and oral function: a systematic review. Int J Oral Maxillofac Surg 2017;46:554-63.

23. Giudice A, Barone S, Belhous K, Morice A, Soupre V, Bennardo F, et al. Pierre Robin sequence: a comprehensive narrative review of the literature over time. $\mathrm{J}$ Stomatol Oral Maxillofac Surg 2018;119:419-28.

24. Nicolielo LFP, Jacobs R, Ali Albdour E, Hoste X, Abeloos J, Politis C, et al. ls oestrogen associated with mandibular condylar resorption? A systematic review. Int J Oral Maxillofac Surg 2017;46:1394402.

25. Wolford LM. Idiopathic condylar resorption of the temporomandibular joint in teenage girls (cheerleaders syndrome). Proc (Bayl Univ Med Cent)
2001;14:246-52.

26. Aneja V, Raval R, Aneja P, Rai KK, Agarwal S, Chuadhary S. Evaluation of mandibular condylar changes in patients following orthognathic surgery: a retrospective study. Niger J Surg 2017;23:37-41.

27. Gunson MJ, Arnett GW, Milam SB. Pathophysiology and pharmacologic control of osseous mandibular condylar resorption. J Oral Maxillofac Surg 2012;70:1918-34.

28. Amarista FJ, Mercuri LG, Perez D. Temporomandibular joint prosthesis revision and/or replacement survey and review of the literature. J Oral Maxillofac Surg 2020;78:1692-703.

29. Ha MH, Kim Yl, Park SB, Kim SS, Son WS. Conebeam computed tomographic evaluation of the condylar remodeling occurring after mandibular setback by bilateral sagittal split ramus osteotomy and rigid fixation. Korean J Orthod 2013;43:263-70.

30. Xi T, Schreurs R, van Loon B, de Koning M, Bergé S, Hoppenreijs T, et al. 3D analysis of condylar remodelling and skeletal relapse following bilateral sagittal split advancement osteotomies. J Craniomaxillofac Surg 2015;43:462-8. 